\title{
An Algorithm for Correcting Levels of Useful Signals on Interpretation of Eddy-Current Defectograms
}

\author{
E. V. Kuzmin ${ }^{1}$, O. E. Gorbunov², P. O. Plotnikov ${ }^{2}$, V. A. Tyukin ${ }^{2}$, V. A. Bashkin ${ }^{1,2}$ \\ DOI: $10.18255 / 1818-1015-2021-1-74-88$
}

${ }^{1}$ P. G. Demidov Yaroslavl State University, 14 Sovetskaya str., Yaroslavl 150003, Russia.

${ }^{2}$ Center of Innovative Programming, NDDLab, 144 Soyuznaya str., Yaroslavl 150008, Russia.

MSC2020: 68T09

Research article

Full text in Russian
Received October 21, 2020

After revision February 17, 2021

Accepted March 12, 2021

To ensure traffic safety of railway transport, non-destructive tests of rails are regularly carried out by using various approaches and methods, including eddy-current flaw detection methods. An automatic analysis of large data sets (defectograms) that come from the corresponding equipment is an actual problem. The analysis means a process of determining the presence of defective sections along with identifying structural elements of railway tracks in defectograms. This article continues the cycle of works devoted to the problem of automatic recognizing images of defects and structural elements of rails in eddy-current defectograms. In the process of forming these images, only useful signals are taken into account, the threshold levels of amplitudes of which are determined automatically from eddy-current data. The previously used algorithm for finding threshold levels was focused on situations in which the vast majority of signals coming from the flaw detector is a rail noise. A signal is considered useful and is subject to further analysis if its amplitude is twice the corresponding noise threshold. The article is devoted to the problem of correcting threshold levels, taking into account the need to identify extensive surface defects of rails. An algorithm is proposed for finding the values of threshold levels of rail noise amplitudes with their subsequent correction in the case of a large number of useful signals from extensive defects. Examples of the algorithm's operation on real eddy-current data are given.

Keywords: nondestructive testing, eddy current testing, rail flaw detection, automated analysis of defectograms

\section{INFORMATION ABOUT THE AUTHORS}

\begin{tabular}{r|l}
$\begin{array}{r}\text { Egor V. Kuzmin } \\
\text { correspondence author }\end{array}$ & $\begin{array}{l}\text { orcid.org/0000-0003-0500-306X. E-mail: kuzmin@uniyar.ac.ru, } \\
\text { kuzminev@nddlab.com } \\
\text { Professor, Doctor of Science. }\end{array}$ \\
Oleg E. Gorbunov & $\begin{array}{l}\text { orcid.org/0000-0001-6274-9971. E-mail: gorbunovoe@nddlab.com } \\
\text { General Director, PhD. }\end{array}$ \\
Petr O. Plotnikov & $\begin{array}{l}\text { orcid.org/0000-0001-5687-7969. E-mail: plotnikovpo@nddlab.com } \\
\text { Production Engineer. }\end{array}$ \\
Vadim A. Tyukin & $\begin{array}{l}\text { orcid.org/0000-0001-9149-7435. E-mail: tyukinva@nddlab.com } \\
\text { Head of Software Development. }\end{array}$ \\
Vladimir A. Bashkin & $\begin{array}{l}\text { orcid.org/0000-0002-2534-1026. E-mail: bashkinva@nddlab.com } \\
\text { Professor, Doctor of Science. }\end{array}$
\end{tabular}

Funding: This work was supported by P. G. Demidov Yaroslavl State University Project № VIP-016.

For citation: E. V. Kuzmin, O. E. Gorbunov, P. O. Plotnikov, V. A. Tyukin, and V. A. Bashkin, "An Algorithm for Correcting Levels of Useful Signals on Interpretation of Eddy-Current Defectograms”, Modeling and analysis of information systems, vol. 28, no. 1, pp. 74-88, 2021.

(c) Kuzmin E. V., Gorbunov O. E., Plotnikov P. O., Tyukin V. A., Bashkin V. A., 2021

This is an open access article under the CC BY license (https://creativecommons.org/licenses/by/4.0/). 


\title{
Алгоритм корректировки уровней полезных сигналов при расшифровке вихретоковых дефектограмм
}

\author{
Е. В. Кузьмин ${ }^{1}$, О. Е. Горбунов ${ }^{2}$, П. О. Плотников ${ }^{2}$, В. А. Тюкин ${ }^{2}$, В. А. Башкин ${ }^{1,2}$ \\ DOI: $10.18255 / 1818-1015-2021-1-74-88$
}

${ }_{1}^{1}$ Ярославский государственный университет им. П. Г. Демидова, ул. Советская, д. 14, г. Ярославль, 150003 Россия. ${ }^{2} \mathrm{OOO}$ «Центр инновационного программирования», NDDLab, ул. Союзная, д. 144, г. Ярославль, 150008 Россия.

УДК 004.021

Научная статья

Полный текст на русском языке
Получена 21 октября 2020 г. После доработки 17 февраля 2021 г. Принята к публикации 12 марта 2021 г.

Для обеспечения безопасности движения на железнодорожном транспорте регулярно проводится неразрушающий контроль рельсов с применением различных подходов и методов, включая методы вихретоковой дефектоскопии. Актуальной задачей является автоматический анализ больших массивов данных (дефектограмм), которые поступают от соответствующего оборудования. Под анализом понимается процесс определения по дефектограммам наличия дефектных участков наряду с выявлением конструктивных элементов рельсового пути. Данная статья продолжает цикл работ, посвященных задаче автоматического распознавания образов дефектов и конструктивных элементов железнодорожных рельсов по вихретоковым дефектограммам. При формировании этих образов принимаются в расчет только полезные сигналы, пороговые уровни амплитуд которых определяются автоматически по вихретоковым данным. Применяемый ранее алгоритм нахождения пороговых уровней был ориентирован на ситуации, при которых подавляющее большинство поступающих от дефектоскопа сигналов составляет рельсовый шум. Сигнал считается полезным и подлежит дальнейшему анализу, если его амплитуда в два раза превосходит соответствующий пороговый уровень шума. Статья посвящена задаче корректировки пороговых уровней с учётом необходимости выявления протяжённых поверхностных дефектов рельсов. Предлагается алгоритм нахождения значений пороговых уровней амплитуд рельсового шума с их последующей корректировкой в случае наличия большого количество полезных сигналов от протяженных дефектов. Приводятся примеры работы алгоритма на реальных вихретоковых данных.

Ключевые слова: неразрушающий контроль рельсов, вихретоковая дефектоскопия, обнаружение дефектов, автоматический анализ дефектограмм

\section{ИНФОРМАЦИЯ ОБ АВТОРАХ}

Егор Владимирович Кузьмин автор для корреспонденции

Олег Евгеньевич Горбунов

Петр Олегович Плотников

Вадим Александрович Тюкин

Владимир Анатольевич Башкин
orcid.org/0000-0003-0500-306X. E-mail: kuzmin@uniyar.ac.ru,

kuzminev@nddlab.com

профессор, доктор физ.-мат. наук.

orcid.org/0000-0001-6274-9971. E-mail: gorbunovoe@nddlab.com генеральный директор, канд. физ.-мат. наук.

orcid.org/0000-0001-5687-7969. E-mail: plotnikovpo@nddlab.com инженер-технолог.

orcid.org/0000-0001-9149-7435. E-mail: tyukinva@nddlab.com руков. сектора разработки.

orcid.org/0000-0002-2534-1026. E-mail: bashkinva@nddlab.com профессор, доктор физ.-мат. наук.

Финансирование: Работа выполнена в рамках инициативной НИР ЯрГУ им. П. Г. Демидова № VIP-016.

Для цитирования: E. V. Kuzmin, O. E. Gorbunov, P. O. Plotnikov, V. A. Tyukin, and V. A. Bashkin, "An Algorithm for Correcting Levels of Useful Signals on Interpretation of Eddy-Current Defectograms”, Modeling and analysis of information systems, vol. 28, no. 1, pp. 74-88, 2021.

( Кузьмин Е. В., Горбунов О. Е., Плотников П. О., Тюкин В. А., Башкин В. А., 2021

Эта статья открытого доступа под лицензией СС BY license (https://creativecommons.org/licenses/by/4.0/). 


\section{Введение}

Для обеспечения безопасности движения на железнодорожном транспорте регулярно проводится неразрушающий контроль рельсов с применением различных подходов и методов, включая методы вихретоковой дефектоскопии. Актуальной задачей является автоматический анализ [1-3] больших массивов данных (дефектограмм), которые поступают от соответствующего оборудования. Под анализом понимается процесс определения по дефектограммам наличия дефектных участков наряду с выявлением конструктивных элементов рельсового пути. При этом в условиях значительных объемов поступающей на обработку информации наибольший интерес представляют быстрые и эффективные алгоритмы анализа данных.

Данная статья продолжает цикл работ [4-7], посвященных задаче автоматического распознавания образов дефектов и конструктивных элементов железнодорожных рельсов по дефектограммам вихретоковых дефектоскопов. Дефектограммы разбиваются на фрагменты (блоки анализа), каждый из которых обрабатывается отдельно. В текущем блоке анализа с использованием алгоритма из статей $[4,5]$ происходит выделение полезных сигналов (на фоне рельсового шума), которые группируются в отметки. Найденные отметки подлежат дальнейшей классификации с применением нейронных сетей. В статье [6] решалась задача распознавания записей небольших конструктивных элементов (длиной до 157 мм) следующих трёх типов: 1) болтовой стык с прямым или скошенным соединением рельсов, 2) электроконтактная сварка рельсов и 3) алюминотермитная сварка рельсов. В статье [7] проводилось распознавание записей длинных (от 420 мм до 3220 мм) конструктивных элементов рельсового пути двух классов: 1) счётчик осей подвижного состава, 2) пересечение рельсовых путей. Отметки, которые не были отнесены к тому или иному типу конструктивных элементов, классифицируются как условные дефекты.

Отметим, что применяемый алгоритм определения порогового уровня амплитуд полезных сигналов $[4,5]$ рассчитан на фрагменты вихретоковых дефектограмм, подавляющее большинство сигналов в которых составляет рельсовый шум. Практическое использование этого алгоритма в рамках аппаратно-программного комплекса рельсовой дефектоскопии показало, что при анализе рельсов с длинными поверхностными дефектами (образующимися обычно из-за контактной усталости металла), которые могут иметь протяжённость в десятки и сотни метров, выдаваемые пороговые значения амплитуд полезных сигналов являются завышенными (по абсолютному значению) и не позволяют формировать в отметки ни записи конструктивных элементов, ни записи дефектных участков рельсов.

Данная статья посвящена задаче корректировки порогового уровня амплитуд полезных сигналов при автоматической расшифровке дефектограмм вихретоковых дефектоскопов с учётом необходимости выявления протяжённых поверхностных дефектов рельсов.

В статье рассматривается обобщённое устройство в виде 12-разрядного вихретокового дефектоскопа с 15 каналами данных (на один рельс). Каналы данных соответствуют физическим датчикам, которые последовательно располагаются на поверхности рельса перпендикулярно направлению движения дефектоскопа. Значения амплитуд сигналов каждого канала регистрируются дефектоскопом в виде целых чисел от -2048 до 2047.

Интерес представляют амплитуды только полезных сигналов. Сигнал считается полезным (и подлежит дальнейшему анализу), если отклонение от нуля как минимум в два раза превосходит соответствующий пороговый уровень шума рельсов.

В статье предлагается алгоритм нахождения положительного и отрицательного значений пороговых уровней амплитуд рельсового шума с их последующей корректировкой в сторону нуля в случае наличия большого количества полезных сигналов (например, от протяженных поверхностных дефектов рельсов) на анализируемом фрагменте вихретоковой дефектограммы; приводятся примеры работы алгоритма на реальных данных. 


\section{1. Нахождение пороговых уровней полезных сигналов}

При автоматическом анализе дефектограммы обычно разбиваются на фрагменты, которые, например, могут соответствовать 50-метровым участкам рельсового пути, т. е. при снятии показаний 15-канального дефектоскопа с каждого миллиметра пути блок анализа представляет собой матрицу размером 15 строк на 50000 столбцов, где элемент матрицы - это значение амплитуды сигнала соответствующего канала данных.

Пример графического представления 50-метрового блока анализа приведён на рис. 1 (15-канальные данные с 50-метрового участка одного рельса; нумерация каналов данных производится сверху вниз). В этом фрагменте дефектограммы помимо записей конструктивных элементов (сварных рельсовых стыков), сливающихся на рисунке в сплошные вертикальные линии, можно видеть, что начиная приблизительно с 18-го метра и до конца рассматриваемого фрагмента присутствуют сильные полезные сигналы от продолжительного поверхностного дефекта.

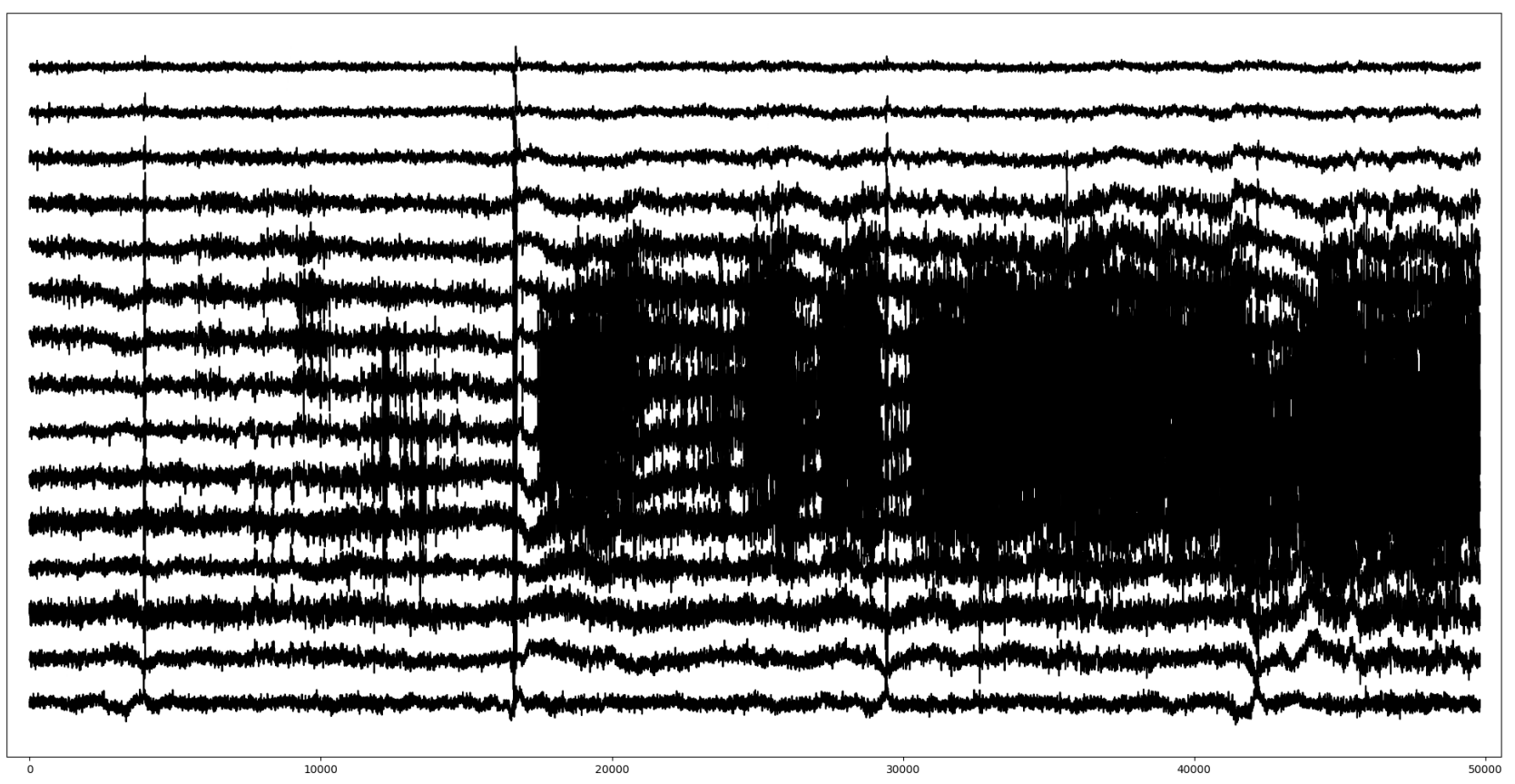

Fig. 1. Eddy-current data (15 channels). An example of an analysis block (50 $\mathrm{m})$

Рис. 1. Вихретоковые данные по 15 каналам. Пример 50-метрового блока анализа

При этом первые три канала вихретоковых данных никак не захватывают этот дефект, регистрируя главным образом рельсовый шум. Основной объём сигналов от дефекта приходится на центральные каналы. В качестве примера на рис. 2 показаны отдельно данные седьмого и восьмого каналов, которые содержат наиболее сильные сигналы от дефекта (т. е. наиболее сильные амплитудные отклонения от нуля).

Приведённый пример специально подобран таким образом, чтобы было видно отличие участков рельсового шума от участков продолжительного дефекта поверхности катания. Дефект может охватывать всю поверхность рельса на протяжении нескольких сотен метров. В таком случае могут возникнуть трудности в визуальном восприятии того, содержит ли дефектограмма запись дефекта или же это рельсовый шум, который был зарегистрирован датчиками с некорректными настройками чувствительности. 

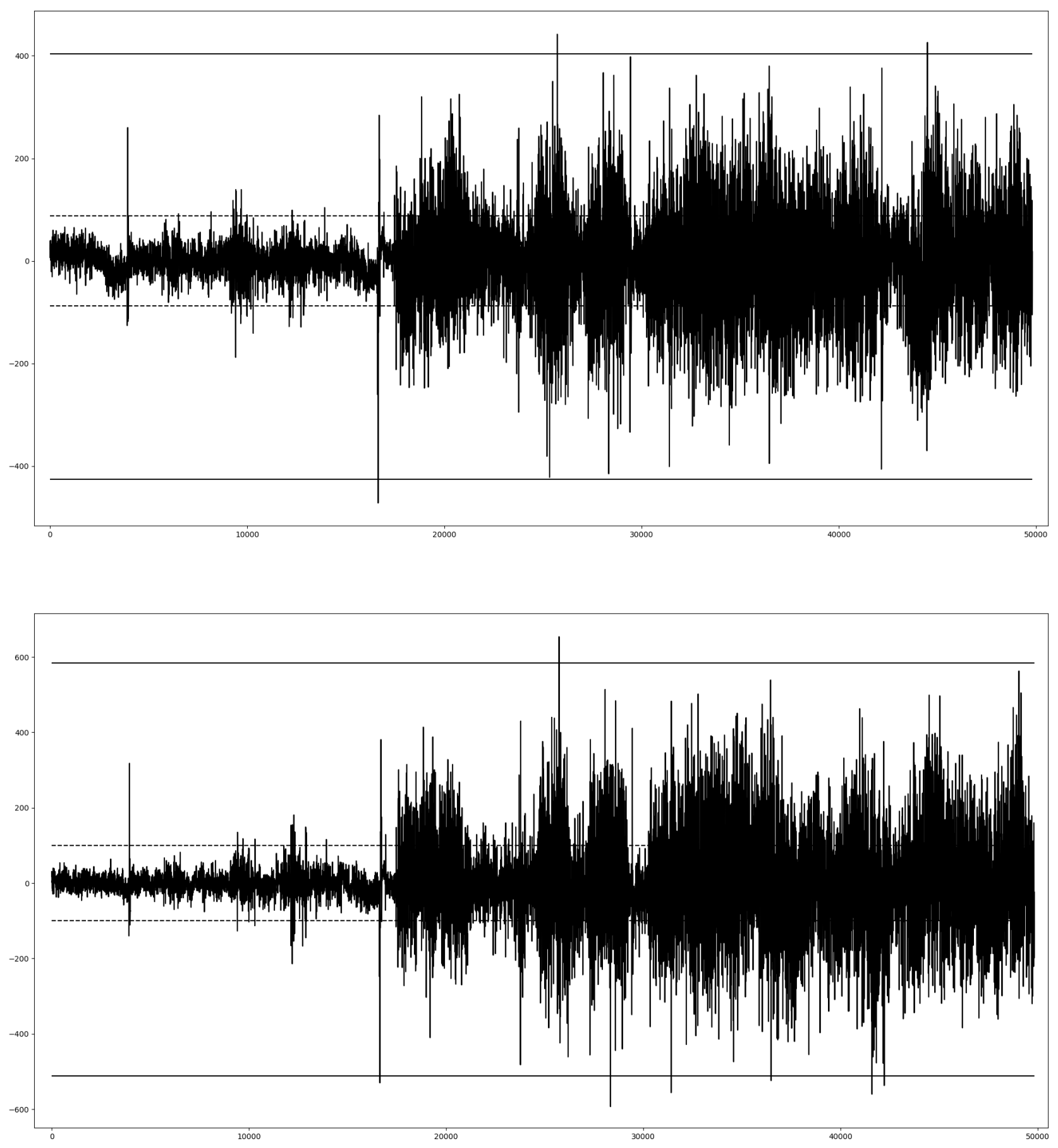

Fig. 2. Eddy-current data of the seventh (top) and the eighth (bottom) channels (50-meter analysis block). Useful signal levels before (solid lines) and after (dashed lines) correcting
Рис. 2. Вихретоковые данные 50-метрового блока анализа по седьмому (вверху) и восьмому (внизу) каналам. Уровни полезных сигналов до (сплошные линии) и после (пунктирные линии) корректировки 
В одном блоке анализа для каждого канала данных отдельно вычисляются положительное и отрицательное пороговые значения амплитуд сигналов рельсового шума. При вычислении положительного порога принимаются во внимание только неотрицательные амплитуды сигналов. Аналогичным образом для вычисления отрицательного порога берутся в расчет только те амплитуды, значение которых меньше или равно нулю.

Следующая функция на языке Python 3 возвращает абсолютное значение искомого порога рельсового шума для одного канала данных. В этой функции параметр $A[0: 2047]$ - это вспомогательный массив, который хранит частоты соответствующие его индексам амплитуд сигналов одной направленности (положительной или отрицательной). Элемент $A[k]$ представляет собой количество сигналов (одной направленности) с амплитудой $k$ по абсолютному значению. Параметр end - максимальное абсолютное значение амплитуды рассматриваемой направленности, т. е. максимальный задействованный индекс массива $A$, a total - сумма значений элементов массива $A$.

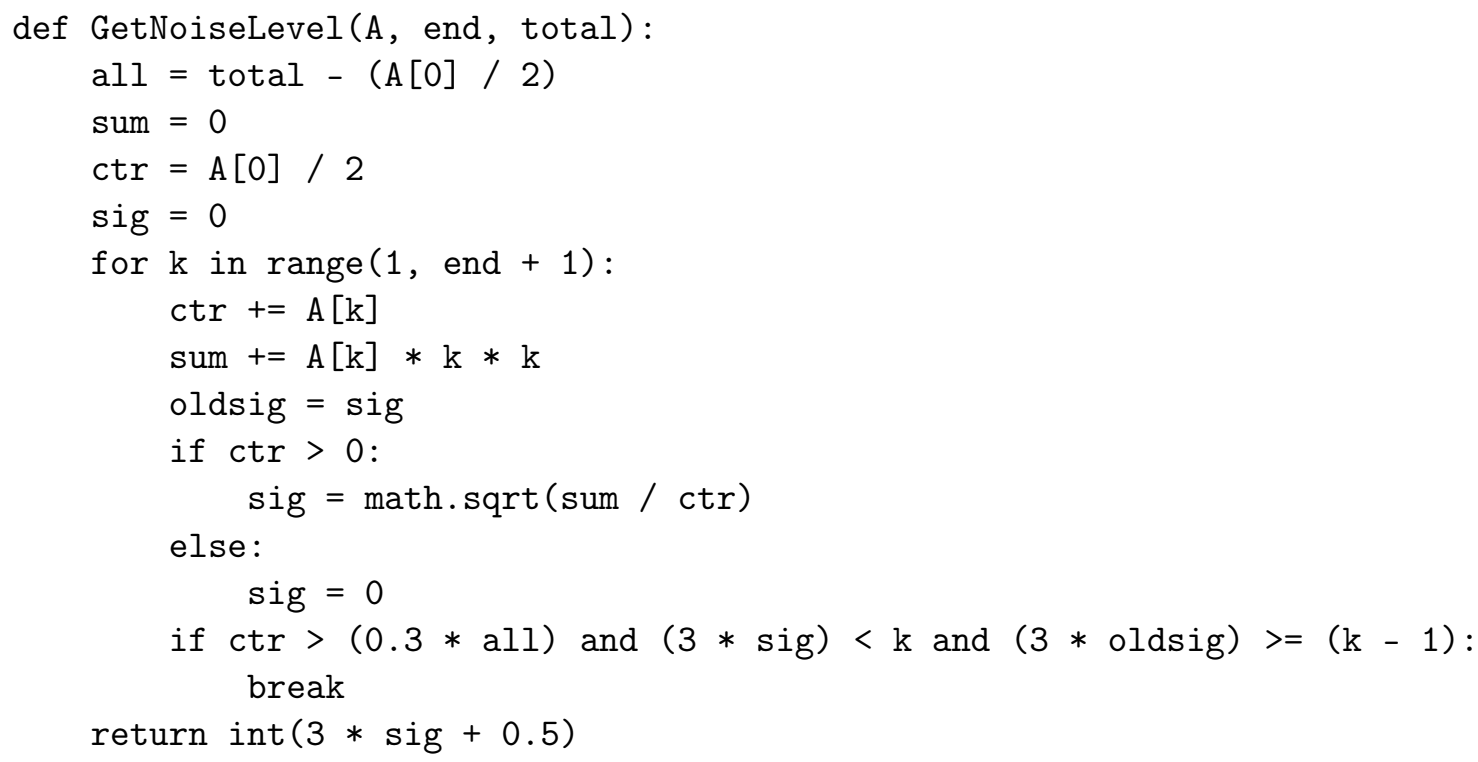

Эта функция реализует алгоритм, предложенный и обоснованный в статьях [4, 5]. Идея алгоритма основывается на том, что вероятность появления сигнала с некоторой амплитудой в бездефектных рельсах на участке без конструктивных элементов подчиняется (в приближении) закону нормального распределения. При этом предполагается, что такие сигналы, которые по своей сути являются рельсовым шумом, будут составлять подавляющее большинство от общего числа сигналов, поступающих от вихретокового дефектоскопа при неразрущающем контроле рельсов. В таком случае может быть задействовано правило трёх сигм с предварительным исключением из анализируемой выборки сильных сигналов от дефектов и конструктивных элементов, которые не укладываются в рамки закона нормального распределения.

Практика показала, что при появлении на дефектограммах записей продолжительных поверхностных дефектов этот алгоритм выдаёт завышенные (по абсолютному значению) амплитудные пороги рельсового шума. Пороговые уровни полезных сигналов также оказываются завышенными, поскольку представляют собой удвоение найденных пороговых значений рельсового шума.

Например, на рис. 2 вычисленные с использованием указанного алгоритма положительный и отрицательный пороговые уровни полезных сигналов для седьмого и восьмого канала данных представлены в виде сплошных линий отсечки. Здесь почти все сигналы от конструктивных элементов и дефектов находятся между найденными пороговыми значениями, т. е. эти сигналы не будут учтены в последующих алгоритмах анализа текущего фрагмента дефектограммы. 

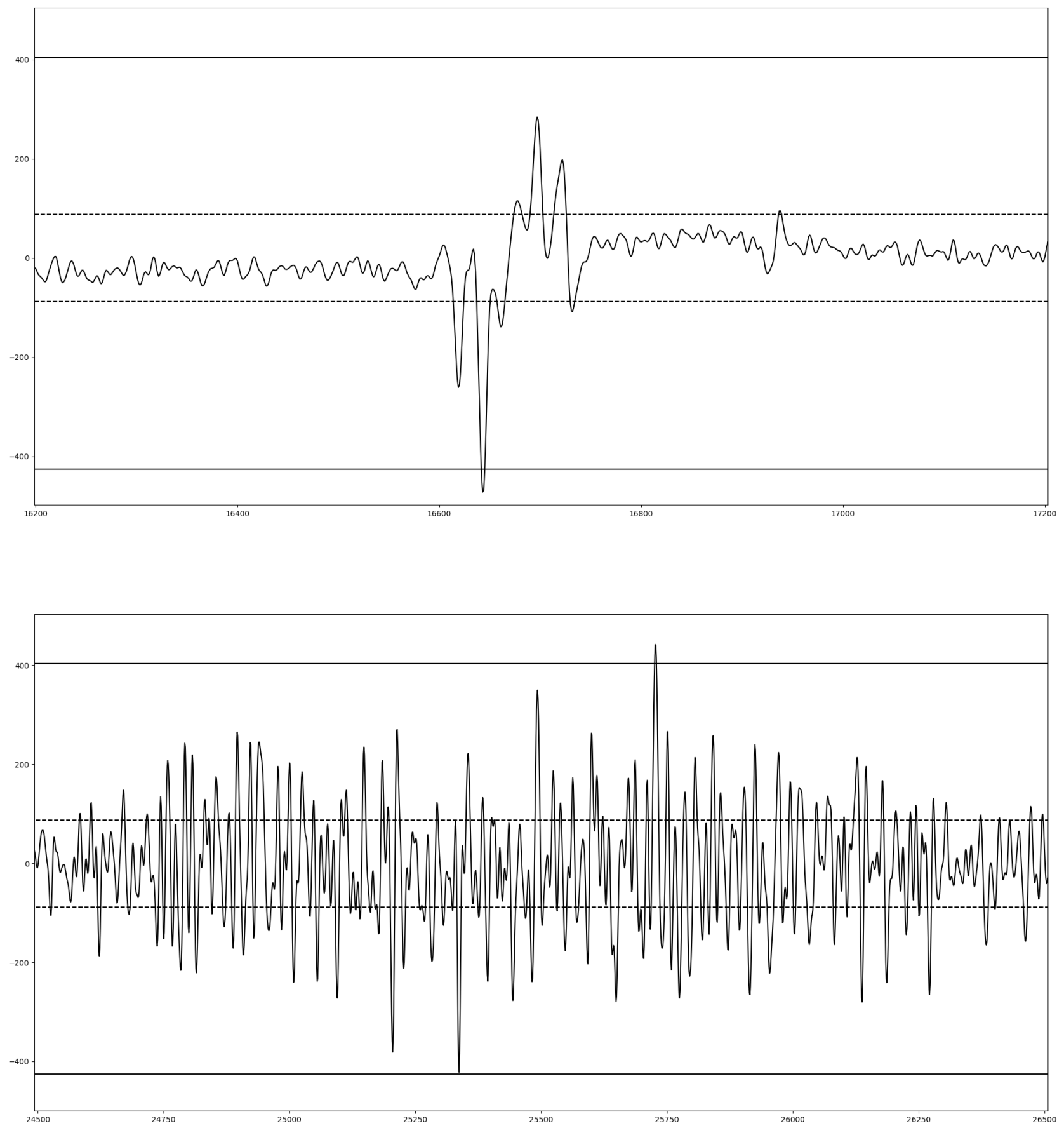

Fig. 3. Eddy-current data of the seventh channel. The aluminothermic weld (top) and the surface defect (bottom). Useful signal levels before (solid lines) and after (dashed lines) correcting
Рис. 3. Вихретоковые данные по седьмому каналу. Алюминотермитная сварка (вверху) и поверхностный дефект (внизу). Уровни полезных сигналов до (сплошные линии) и после (пунктирные линии) корректировки 

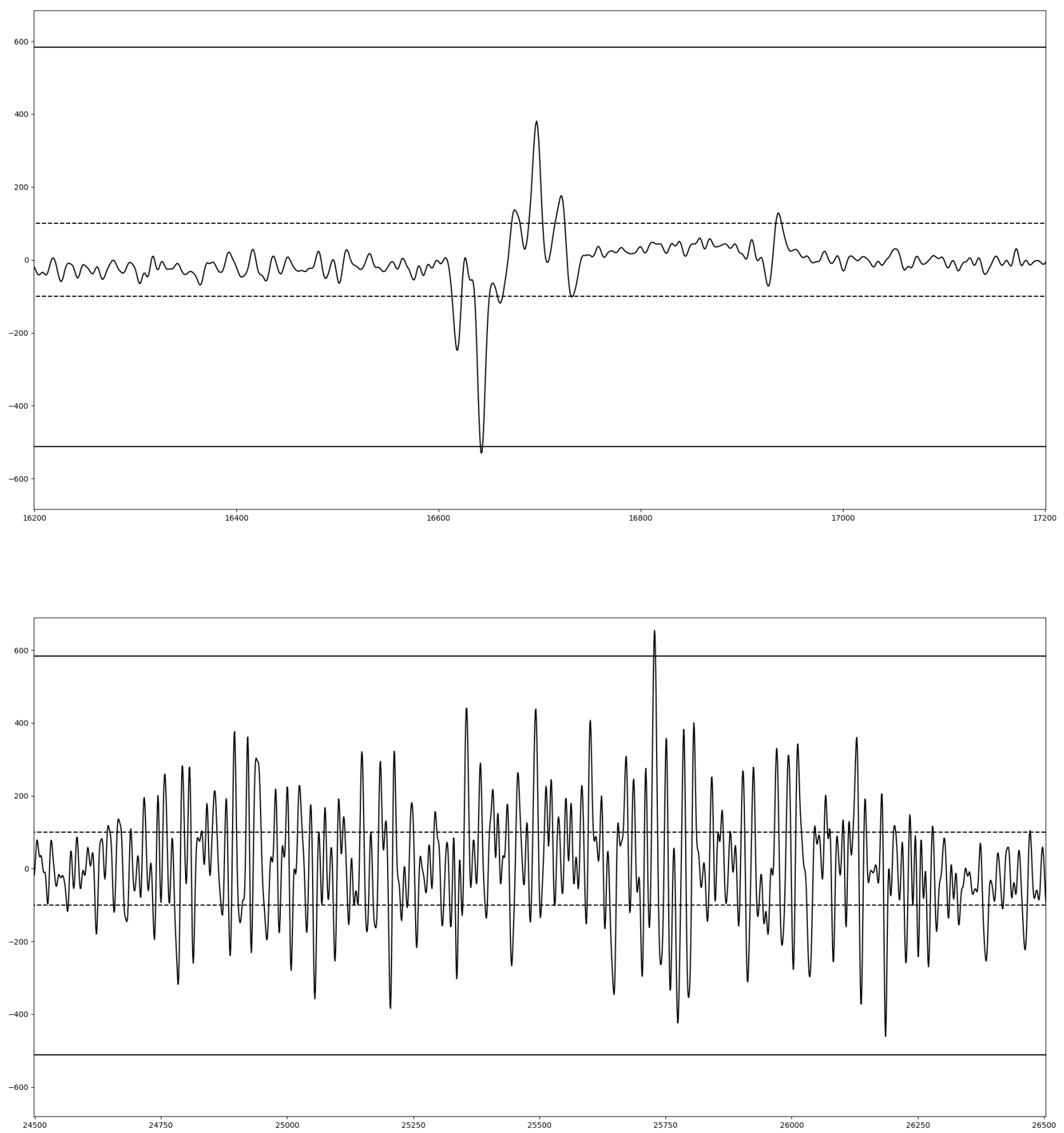

Fig. 4. Eddy current data of the eighth channel. The aluminothermic weld (top) and the surface defect (bottom). Useful signal levels before (solid lines) and after (dashed lines) correcting
Рис. 4. Вихретоковые данные по восьмому каналу. Алюминотермитная сварка (вверху) и поверхностный дефект (внизу). Уровни полезных сигналов до (сплошные линии) и после (пунктирные линии) корректировки 

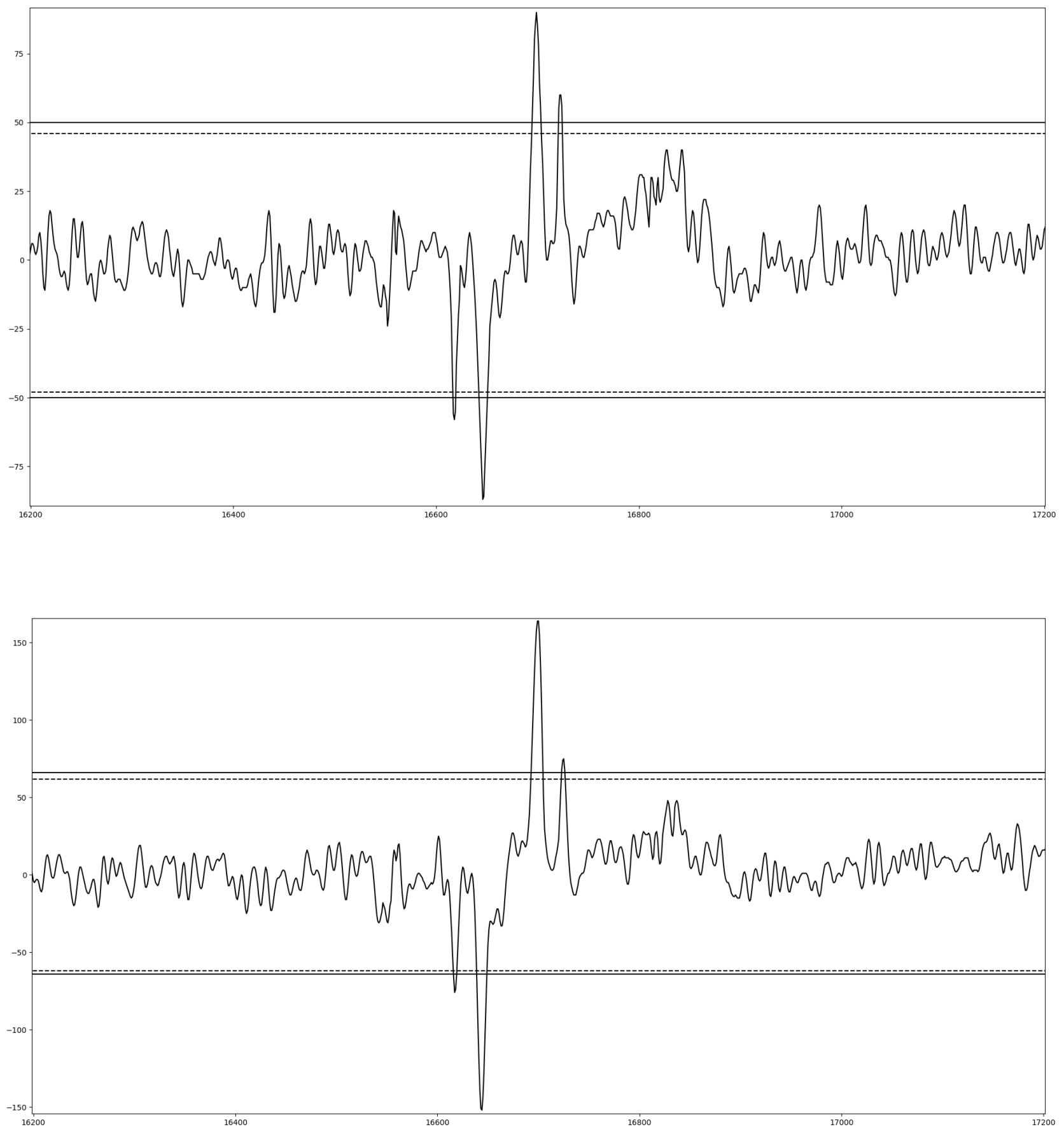

Fig. 5. Eddy current data of the first (top) and the second (bottom) channels. The aluminothermic weld. Useful signal levels before (solid lines) and after (dashed lines) correcting
Рис. 5. Вихретоковые данные по первому (вверху) и второму (внизу) каналам. Алюминотермитная сварка. Уровни полезных сигналов до (сплошные линии) и после (пунктирные линии) корректировки 
На рис. 3 и 4 для седьмого и восьмого каналов данных показаны отдельно участки дефектограммы, содержащие запись сварного алюминотермитного рельсового стыка (вверху) и запись сигналов от поверхностного дефекта (внизу). На этих рисунках найденные пороговые уровни полезных сигналов показаны также в виде сплошных линий отсечки.

Заметим, что первыми двумя каналами дефектоскопа наличие каких-либо поверхностных дефектов на рассматриваемом отрезке рельсового пути не регистрируется (см. рис. 1). Полезными сигналами по этим двум каналам выступают только сигналы от сварных рельсовых стыков. Остальные данные представляют собой рельсовый шум. И как можно видеть на рис. 5, в этом случае сплошные линии найденных пороговых уровней довольно точно отсекают полезные сигналы, поступившие от алюминотермитной сварки. Другими словами, для этих каналов алгоритм нахождения пороговых значений рельсового шума отработал успешно (в соответствии с заложенной в него идеей).

Таким образом, если канал данных текущего блока анализа содержит запись продолжительного поверхностного дефекта, то найденные пороговые значения рельсового шума нуждаются в корректировке в сторону нуля. Решению задачи корректировки этих пороговых уровней посвящён следующий раздел.
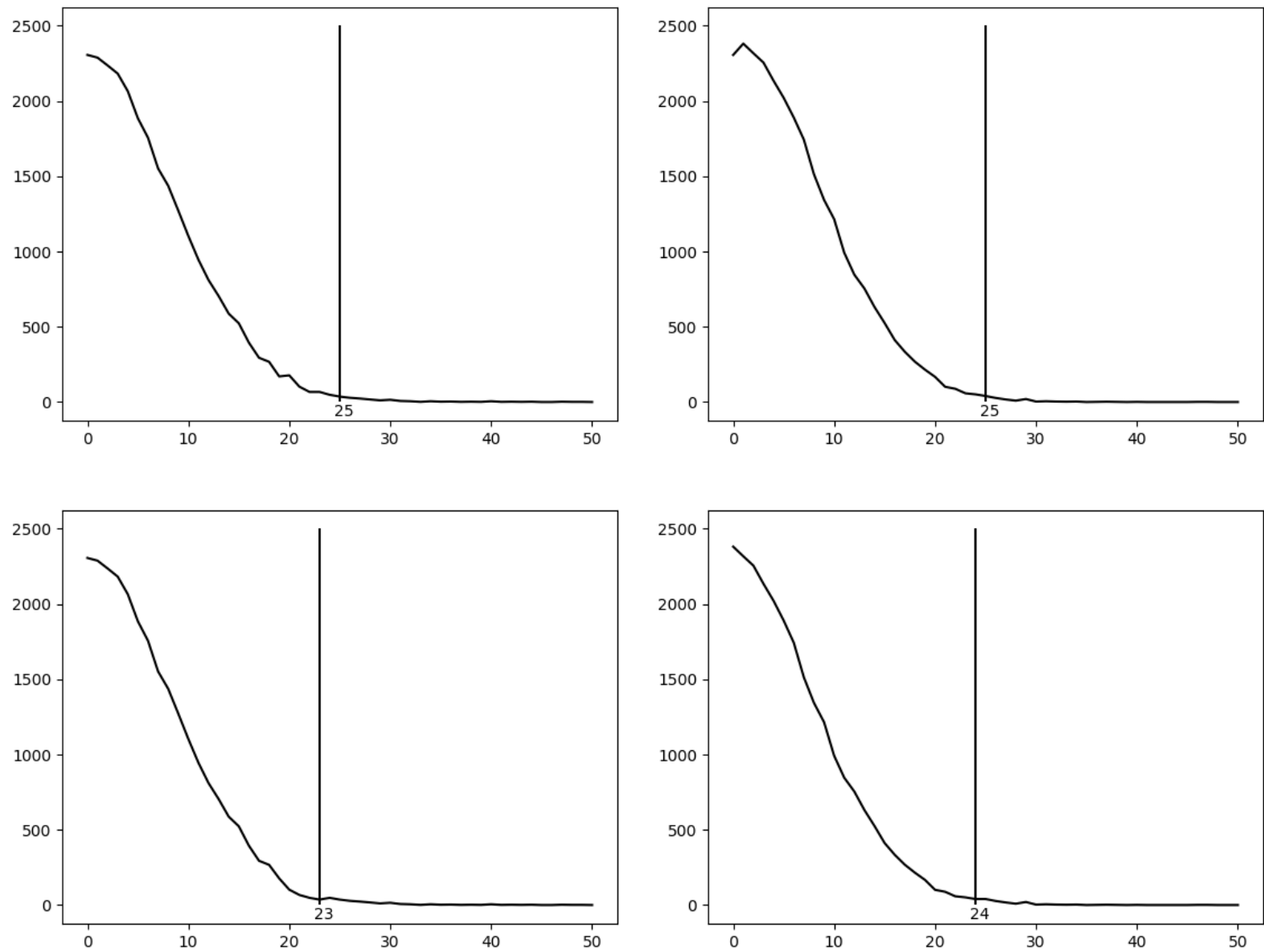

Fig. 6. Graphs of the density of the amplitude frequencies distribution for the first channel before (top) and after (bottom) correcting the noise threshold levels

Рис. 6. Графики плотности распределения частот амплитуд первого канала до (вверху) и после (внизу) корректировки пороговых уровней шума 


\section{2. Корректировка пороговых уровней полезных сигналов}

Практический опыт работы с вихретоковыми данными позволил выявить характерный признак наличия на записи сигналов от продолжительного поверхностного дефекта.

В блоке анализа для одного канала, содержащего сигналы от такого дефекта, на графике плотности распределения частот появления амплитуд одной направленности на отрезке от нуля до порогового значения рельсового шума будут находиться точки локальных минимумов, количество которых зависит от степени опасности поверхностного дефекта, т. е. от количества полезных сигналов и значений их амплитуд. Для «бездефектного» канала данных этот график на том же отрезке будет значительно более гладким, в идеале не имея ни одного локального минимума. При этом в любом случае форма графика на указанном отрезке повторяет в приближении форму графика плотности нормального распределения вероятностей на участке от 0 до $3 \cdot \sigma$, где $\sigma-$ среднеквадратическое отклонение от нулевого математического ожидания.

Локальные минимумы представляют интерес как ресурс, который может быть использован для уменьшения абсолютного значения порогового уровня рельсового шума. Итерационное удаление точек локальных минимумов на рассматриваемом отрезке графика с последующим сдвигом остальных данных в сторону нуля позволяет произвести также и сдвиг порогового уровня шума. Процедура останавливается, как только на графике от нуля до текущего значения порога не останется ни одного локального минимума. Получившееся в результате таких сдвигов новое значение порогового уровня и будет искомым результатом, т. е. будет являться скорректированным порогом рельсового шума.

Следующая функция на языке Python 3 реализует описанную идею, возвращая в качестве результата скорректированный пороговый уровень. Здесь параметр $A[0: 2047]$, как и ранее, представляет собой массив (график) частот амплитуд сигналов одной направленности, т. е. $A[k]-$ это количество сигналов с амплитудой $k$ по абсолютному значению. Параметр level - найденный предыдущим алгоритмом пороговый уровень шума (для амплитуд одной направленности), который подлежит корректировке.

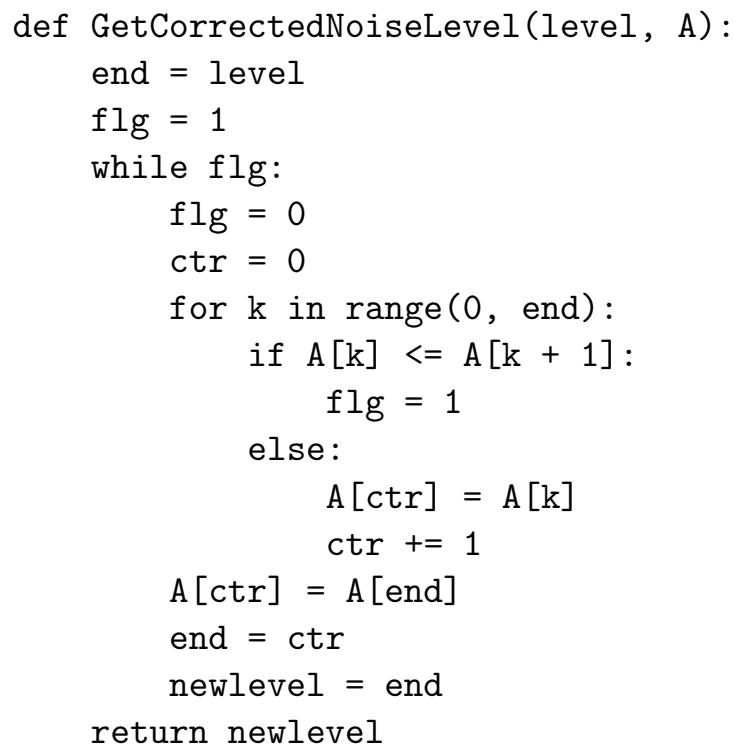

На рис. 6, 7, 8 и 9 представлены графики плотности распределения частот положительных (справа) и отрицательных (слева) амплитуд сигналов первого, второго, седьмого и восьмого каналов данных до (сверху) и после (снизу) корректировки порогового уровня рельсового шума, который отмечен вертикальной линией отсечки с указанием его абсолютного значения. 

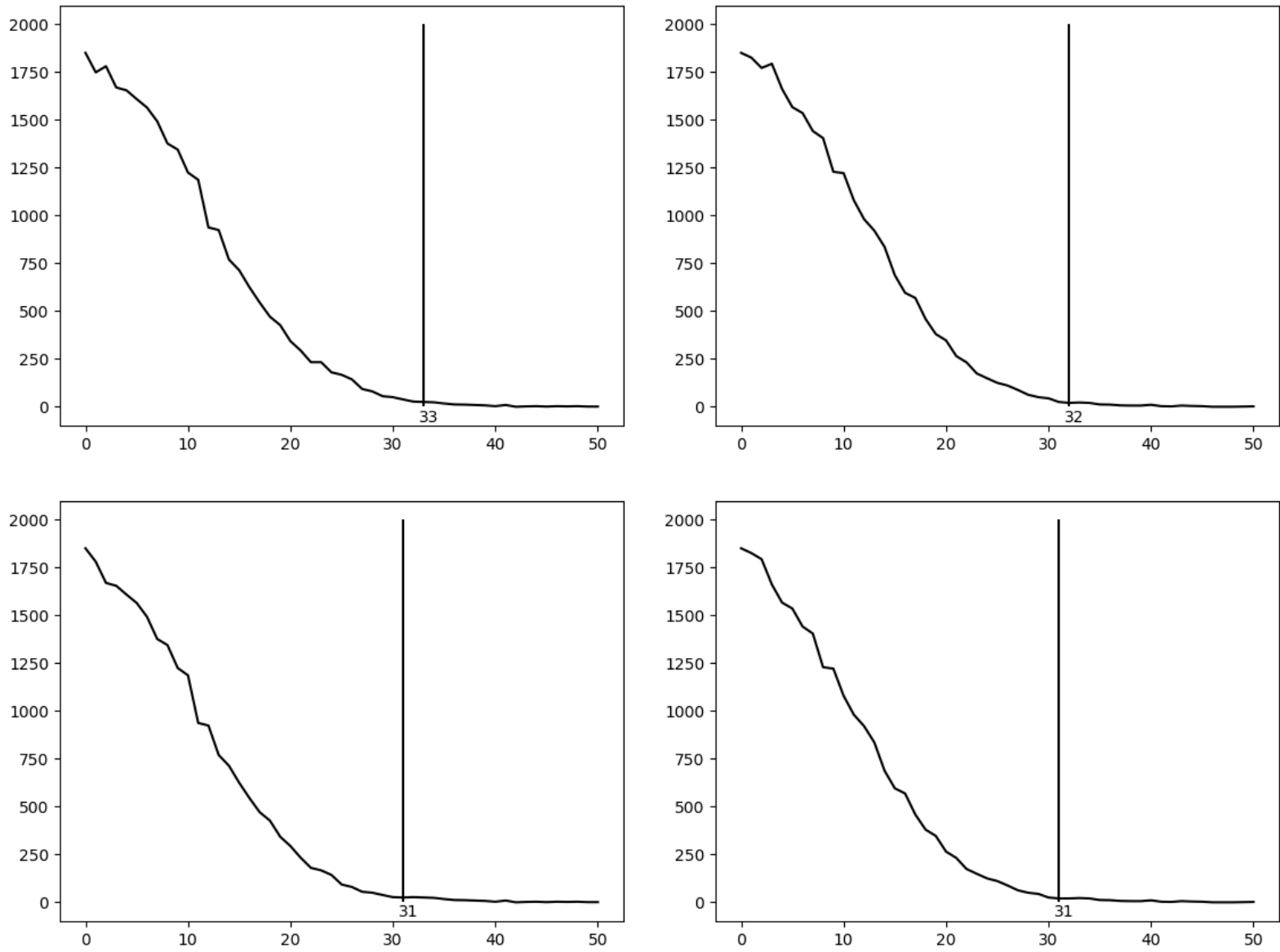

Fig. 7. Graphs of the density of the amplitude frequencies distribution for the second channel before (top) and after (bottom) correcting the noise threshold levels

Рис. 7. Графики плотности распределения частот амплитуд второго канала до (вверху) и после (внизу) корректировки пороговых уровней шума

На этих рисунках для канала данных текущего блока анализа значения амплитуд сигналов одинаковой направленности откладываются по оси X, а суммарное количество зарегистрированных сигналов одной амплитуды отмечается по оси Ү.

На рис. 6 и 7 видно, что графики распределения частот амплитуд сигналов являются «гладкими», почти не имеют точек локальных минимумов. И, соответственно, для этих каналов произведена незначительная корректировка пороговых уровней.

Исходные графики частот амплитуд на рис. 8 и 9 содержат большое количество локальных минимумов и подвергаются значительному сжатию, приближённо сохраняя в результате сглаживания начальную форму на отрезке от нуля до отметки порогового уровня.

Результат работы алгоритма корректировки продемонстрирован на рис. 2, 3 и 4. Новый положительный/отрицательный пороговый уровень амплитуд полезных сигналов, равный положительному/отрицательному удвоенному скорректированному порогу шума, показан в виде пунктирной линии отсечки. На этих рисунках значения полезных сигналов от сварных стыков, в частности от алюминотермитной сварки, и продолжительного поверхностного дефекта выходят за границы, обозначенные пунктирными линиями. Это означает, что такие сигналы впоследствии будут участвовать в формировании соответствующих отметок как для конструктивных элементов, так и для участка продолжительного дефекта поверхности катания рельсов. 

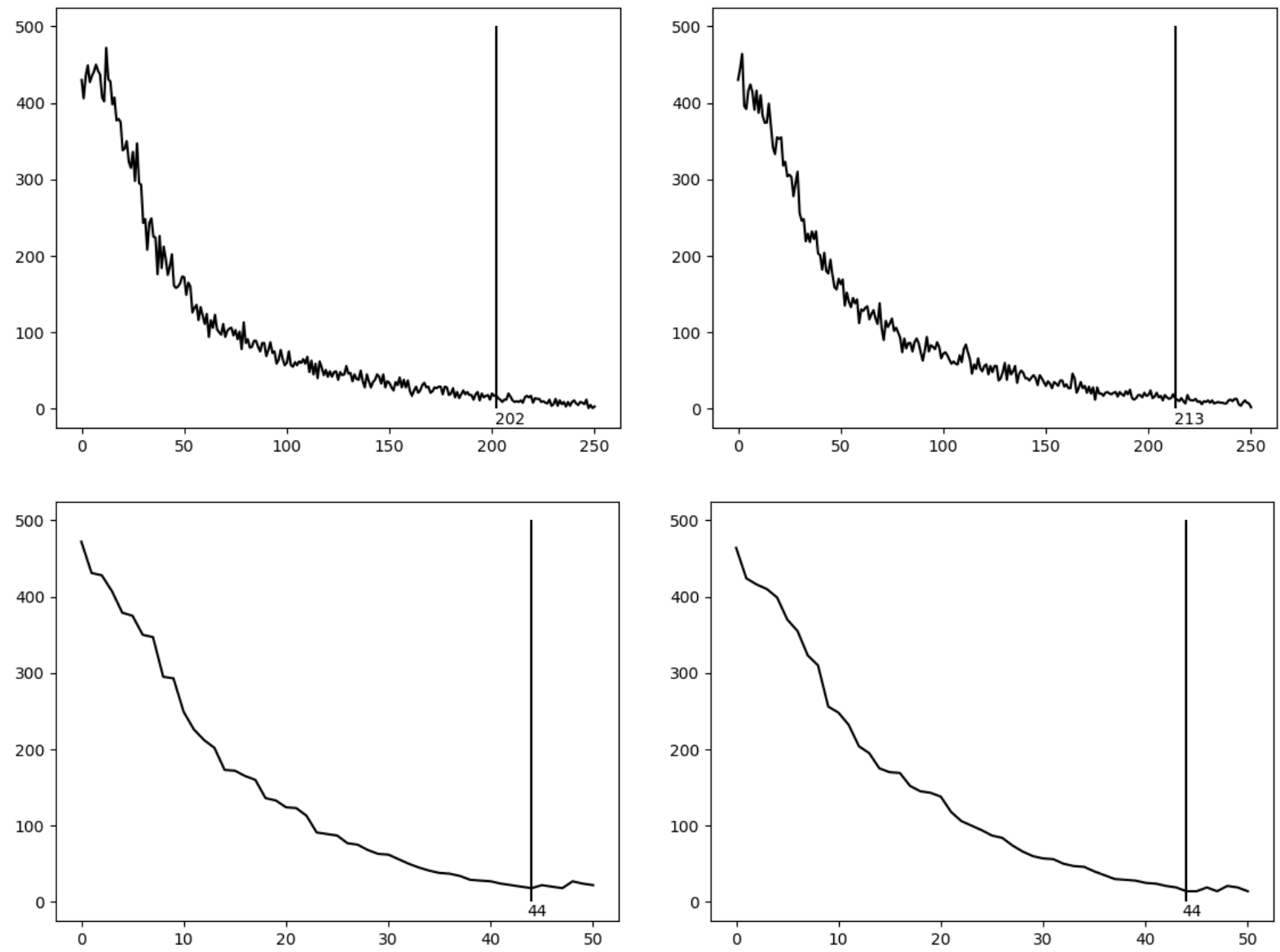

Fig. 8. Graphs of the density of the amplitude frequencies distribution for the seventh channel before (top) and after (bottom) correcting the noise threshold levels

Рис. 8. Графики плотности распределения частот амплитуд седьмого канала до (вверху) и после (внизу) корректировки пороговых уровней шума

Отметим, что на основе скорректированных пороговых уровней также будет производиться и оценка степени опасности найденного поверхностного дефекта. Вопросы оценки степени опасности выделенных на дефектограмме дефектов в данной статье не рассматриваются.

В случае с первым и вторым каналами данных ситуация с пороговыми уровнями полезных сигналов кардинально не изменилась. На рис. 5 пунктирная линия нового порога находится рядом со сплошной линией соответствующего исходного порогового уровня, отсекая всё те же полезные сигналы, поступившие от сварного алюминотермитного рельсового стыка.

Таким образом, предложенный в статье алгоритм корректировки порогового уровня шума может быть применён для любого вихретокового канала без предварительного анализа данных на предмет присутствия сигналов от продолжительного поверхностного дефекта. Корректировка порогов будет проведена по необходимости в зависимости от количества точек локальных минимумов на графиках плотности распределения частот амплитуд.

Алгоритм гарантированно останавливается. Полученный в результате пороговый уровень сохраняет прежний смысл, отмечая на сжатом (сглаженном) графике плотности распределения частот границу амплитуд рельсового шума. 

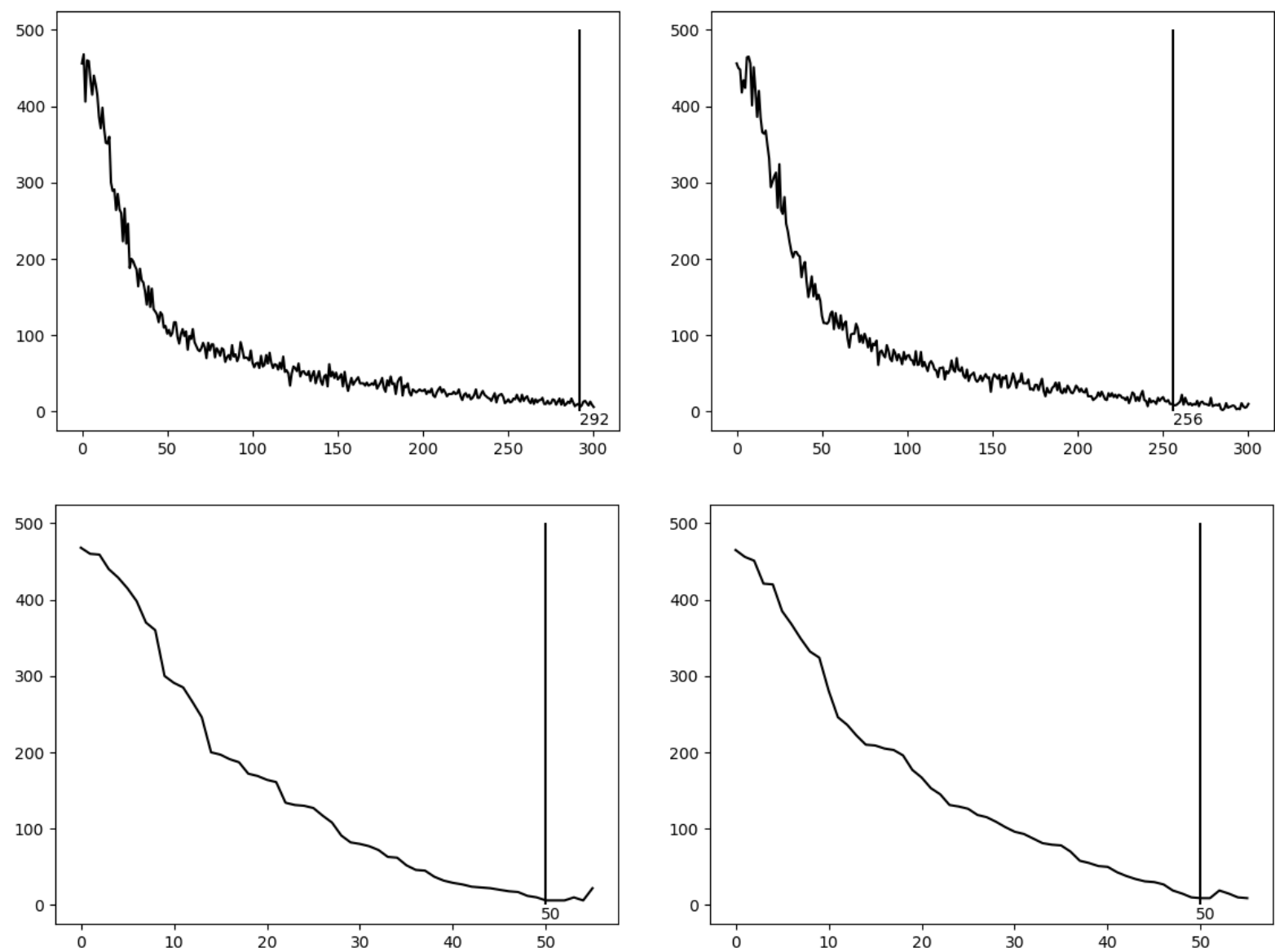

Fig. 9. Graphs of the density of the amplitude frequencies distribution for the eighth channel before (top) and after (bottom) correcting the noise threshold levels

Рис. 9. Графики плотности распределения частот амплитуд восьмого канала до (вверху) и после (внизу) корректировки пороговых уровней шума

Сжатый график на отрезке от нуля до порогового значения в приближении повторяет форму графика плотности нормального распределения вероятностей. Если запустить рассмотренный в предыдущем разделе алгоритм определения порогового уровня шума на этом фрагменте сжатого графика, будут выданы сопоставимые результаты. Например, для седьмого канала оба искомых уровня (и положительный, и отрицательный) будут иметь одинаковое абсолютное значение, равное 47 (найденные алгоритмом корректировки пороговые уровни имели значение 44).

Идея алгоритма основывается на результатах наблюдений, полученных на практике при обработке большого массива вихретоковых данных, а сам алгоритм корректировки приводится без строгого математического обоснования. В связи с этим представляет интерес построение математической модели влияния протяжённых поверхностных дефектов на плотность распределения частот амплитуд сигналов, зафиксированных на дефектограмме.

\section{Заключение}

Предложенный в статье алгоритм корректировки пороговых уровней амплитуд полезных сигналов показал свою эффективность на практике при неразрушающем контроле рельсов. В рамках аппаратно-программного комплекса вихретоковой дефектоскопии алгоритм успешно применяется для выделения сигналов, поступающих от протяжённых дефектов поверхности катания рельсов. 


\section{References}

[1] A. A. Markov and E. A. Kuznetsova, Rails flaw detection. Formation and analysis of signals. Book 1. Principles. St. Petersburg: KultInformPress, 2010.

[2] A. A. Markov and E. A. Kuznetsova, Rails flaw detection. Formation and analysis of signals. Book 2. Data interpretation. St. Petersburg: Ultra Print, 2014.

[3] V. F. Tarabrin, A. V. Zverev, O. E. Gorbunov, and E. V. Kuzmin, "About Data Filtration of the Defectogram Automatic Interpretation by Hardware and Software Complex ASTRA”, NDT World, vol. 64, no. 2, pp. 5-9, 2014.

[4] E. V. Kuzmin, O. E. Gorbunov, P. O. Plotnikov, and V. A. Tyukin, "Finding the Level of Useful Signals on Interpretation of Magnetic and Eddy-Current Defectograms", Automatic Control and Computer Sciences, vol. 52, no. 7, pp. 658-666, 2018.

[5] E. V. Kuzmin, O. E. Gorbunov, P. O. Plotnikov, and V. A. Tyukin, "An Efficient Algorithm for Finding the Level of Useful Signals on Interpretation of Magnetic and Eddy Current Defectograms", Automatic Control and Computer Sciences, vol. 52, no. 7, pp. 867-870, 2018.

[6] E. V. Kuzmin, O. E. Gorbunov, P. O. Plotnikov, V. A. Tyukin, and V. A. Bashkin, "Application of Neural Networks for Recognizing Rail Structural Elements in Magnetic and Eddy Current Defectograms”, Automatic Control and Computer Sciences, vol. 53, no. 7, pp. 628-637, 2019.

[7] E. V. Kuzmin, O. E. Gorbunov, P. O. Plotnikov, V. A. Tyukin, and V. A. Bashkin, "Application of Convolutional Neural Networks for Recognizing Long Structural Elements of Rails in Eddy-Current Defectograms”, Modeling and analysis of information systems, vol. 27, no. 3, pp. 316-329, 2020. 